Ann. Sci. forest., 1972, 29 (4), 427-450.

\title{
PREMIERS RÉSULTATS CONCERNANT DEUX EXPÉRIENCES D'ASSAINISSEMENT DU SOL SUR PLANTATIONS DE RÉSINEUX
}

\author{
G. LÉVY \\ avec la collaboration technique de M. Guillemette \\ Station de Recherches sur les Sols forestiers et la Fertilisation, \\ Centre national de Recherches forestières, I.N.R.A. \\ Champenoux 54370 Einville
}

\begin{abstract}
RÉSUMÉ
L'une des deux expériences (Essegney) est située sur pseudogley formé sur alluvions de la Moselle recouvertes de limons, l'autre (Évaux-et-Ménil) sur pélosol-pseudogley, dont le substratum est constitué des marnes du keuper recouvertes de 25 à $30 \mathrm{~cm}$ de limons. Les essences testées à Essegney sont l'Épicéa, le Douglas, le Mélèze d'Europe, le Pin Weymouth et le Cyprès de Lawson; seules les trois premières ont été utilisées à Évaux.

Quatre modalités d'assainissement ont été comparées : fossés à écartement de $40 \mathrm{~m}, 20 \mathrm{~m}$ et $10 \mathrm{~m}$ et billons. Les résultats présentés sont ceux enregistrés deux ans après la plantation.

A Essegney, des fossés à écartement de 20 et $10 \mathrm{~m}$ permettent d'abaisser sensiblement le niveau de la nappe; ceci accroît nettement la croissance en hauteur de l'Épicéa ainsi que la reprise et la croissance du Douglas et du Mélèze. Pour le Cyprès de Lawson par contre, les billons constituent le traitement le plus efficace.

A Évaux, seule la constitution de billons permet d'accroître l'épaisseur de sol non engorgé; cela se traduit surtout sur le comportement du Douglas : reprise et croissance en hauteur sont très fortement améliorées.
\end{abstract}

\section{I. - INTRODUCTION}

Le problème de l'utilisation forestière des sols à hydromorphie temporaire (défaut d'infiltration des eaux pluviales) se pose dans de nombreuses régions de notre pays. En effet la nappe limite plus ou moins le développement des racines, ce qui peut avoir pour conséquence en particulier une alimentation déficiente en éléments minéraux et surtout en eau (pendant l'été). Mais l'action néfaste de la nappe varie suivant les essences et probablement le stade de développement de l'arbre.

L'acuité de ce problème est plus ou moins marquée suivant les conditions climatiques, 
topographiques, géologiques et pédologiques. Dans le cadre d'une région climatiquement bien définie, les deux derniers facteurs deviennent primordiaux; d'eux dépend la profondeur d'apparition du «plancher » de la nappe, ainsi que le caractère plus ou moins imperméable de celui-ci, donc l'intensité de l'engorgement.

Parmi les sols hydromorphes proprement dits, c'est-à-dire ceux dans lesquels la nappe affleure à certaines périodes à proximité de la surface, on peut ainsi distinguer dans un but pratique :

- les pélosols, sols peu évolués formés sur roche-mère à texture fortement argileuse (tous les pélosols n'ont d'ailleurs pas de caractères hydromorphes très nettement marqués),

- les pélosols-pseudogleys dans lesquels la roche-mère argileuse est recouverte d'un autre substratum en général limoneux qui ne dépasse pas $30 \mathrm{~cm}$ d'épaisseur environ,

- les pseudogleys-types primaires (le caractère imperméable du «plancher » étant d'origine géologique) ou secondaires (ce caractère provenant d'un lessivage des horizons supérieurs). mètres.

Ce plancher se trouve en général à une profondeur supérieure à une trentaine de centi-

En Lorraine, d'importantes surfaces forestières et même agricoles sont concernées par ce problème. En ce qui concerne les zones forestières, elles sont couvertes en général par un taillis ou un taillis sous futaie à base de Chêne pédonculé de faible rapport et dont la régénération sexuée naturelle laisse fortement à désirer. Les forestiers se trouvent confrontés à différents problèmes qui peuvent se résumer ainsi :

- Faut-il maintenir le Chêne tout en opérant une conversion en futaie ou bien est-il préférable d'effectuer des introductions d'essences et lesquelles?

- Quelle que soit la solution retenue, est-il souhaitable d'intervenir au niveau du sol (fertilisation et surtout assainissement)?

Les réponses seront fonction des conditions de station mais également de l'aspect financier du problème : la conversion du peuplement en futaie de Chêne nécessite des frais importants qui ne pourront être récupérés avant un très long délai; le problème se pose donc d'une manière différente pour l'État ou pour un propriétaire privé.

Afin de tenter de résoudre ces problèmes nous avons entamé une étude générale de la croissance des arbres sur ces sols en Lorraine. Cette étude comprend un certain nombre de projets abordés conjointement. Certains de ces projets abordent l'étude des moyens d'intervention au nivcau du sol. Il a été ainsi prévu la mise en place d'expériences d'assainissement portant sur plusieurs essences et divers types de stations. Ces essais devraient permettre de choisir en connaissance de cause à plus ou moins long terme un type de peuplement et éventuellement d'assainissement pour chaque groupe de stations. Ils doivent nous donner aussi la possibilité d'aborder les questions plus fondamentales telles l'effet du régime de la nappe sur la nutrition et la croissance des arbres ou l'étude des modifications chimiques et physiques du sol qu'est susceptible d'entraîner l'assainissement.

Parmi les deux premières expériences mises en place, l'une est située en forêt communale d'Essegney (Vosges) (forêt appartenant au massif de Charmes), sur pseudogley-type formé sur alluvions anciennes de la Moselle recouvertes d'une couche limoneuse. Dans les stations de 
ce type, en plus de sa régénération difficile, le Chêne n'est pas de très bonne qualité. C'est pourquoi l'on procède souvent à des introductions de résineux en général l'Épicéa commun, essence « passe-partout ». Cette essence a évidemment été retenue dans l'expérience ainsi que quatre autres essences résineuses choisies pour diverses raisons et dont on pourra comparer le comportement à celui de l'Épicéa.

L'autre sise en forêt communale d'Évaux-et-Ménil (Vosges) concerne un pélosolpseudogley. Ce sol est encore plus défavorable que celui d'Essegney : le «plancher » du pseudogley est plus proche de la surface, ce qui se traduit par une nappe remontant plus superficiellement et une plus longue durée d'engorgement de la couche de sol théoriquement la plus favorable aux racines (les 30 premiers centimètres) (LÉvy 1969). La production des peuplements naturels est très faible et l'introduction de l'Épicéa commun sans assainissement fournit souvent des résultats médiocres. Pour des raisons matérielles, seules trois essences ont été retenues pour cette expérience.

Les deux expériences sont situées à proximité (moins de $10 \mathrm{~km}$ en ligne droite) l'une de l'autre; le climat est identique; de plus elles ont été plantées en même temps. Tout cela rendra plus intéressante la comparaison des résultats.

Les résultats présentés dans ce mémoire concernent le comportement initial des essences testées, c'est-à-dire reprise et croissance à la fin de la deuxième année de plantation. Ces données sont évidemment très provisoires et ce n'est qu'après de longues années que l'on pourra conclure définitivement. Cependant elles sont loin d'être dénuées d'intérêt en raison de la grande importance que revêt pour le forestier le comportement initial d'une plantation.

\section{II. - MÉTHODES \\ 2.1. - Les stations}

Leurs caractéristiques climatiques générales sont les suivantes : pluviométrie moyenne d'environ $730 \mathrm{~mm}$ par an avec un minimum en hiver et un maximum en juin-juillet mais ces extrêmes sont peu marqués. La température annuelle moyenne est de 9 à $10^{\circ} \mathrm{C}$.

\subsection{Essegney.}

La pente du terrain est très faible, environ $2 \mathrm{p}$. mille, et à peu près uniforme.

La roche-mère est formée d'alluvions anciennes de la Moselle recouvertes par des limons et plus ou moins mélangées à eux dans les horizons superficiels.

Le sol (tableau 1) est un pseudogley typique. Génétiquement il s'agit probablement d'un sol lessivé à pseudogley dont 1 'horizon $A_{2}$ a été partiellement tronqué. Le profil moyen peut être décrit de la façon suivante :

- l'horizon $A_{1}$ est épais $(25$ à $30 \mathrm{~cm}$ ) noirâtre, relativement hydromorphe, son $\mathrm{C} / \mathrm{N}$ est assez élevé (23). C'est un hydromoder acide. La limite avec $A_{2} g$ est nette.

- l'horizon $A_{2} g$ contient dans une matrice de teinte claire d'assez nombreuses taches rouille d'hydromorphie. Il se poursuit environ jusqu'à $-40 \mathrm{~cm}$.

Après un horizon intermédiaire $\mathrm{A}_{2} \mathrm{~g} / \mathrm{Bg}$, apparaît vers - 45 à $-50 \mathrm{~cm}$ en moyenne l'horizon $\mathrm{Bg}$; c'est le plancher du pseudogley; dans une matrice de couleur vive se trouvent de nombreuses traînées blanchâtres à bordures rouille, à direction souvent voisine de la verticale. Cet horizon est nettement plus tassé que les précédents.

La teneur en éléments nutritifs est assez faible dans les horizons de surface et le taux de saturation du complexe absorbant est très faible ce qui risque d'affecter les potentialités nutritives du sol.

La texture d'abord limono-argilo-sableuse, devient argilo-limoneuse en $\mathrm{Bg}$. 
TABLEAU 1 - TABLE 1

Analyses du sol d'Essegney

Analysis of Essegney soil

\begin{tabular}{|c|c|c|c|c|c|c|c|c|c|c|c|c|c|c|c|c|}
\hline Hor. & $\begin{array}{l}\text { Mat. organique } \\
\text { Org. matter } \\
\text { p. cent }\end{array}$ & $\begin{array}{l}\text { C } \\
\text { p. cent }\end{array}$ & $\begin{array}{l}\mathrm{N} \\
\text { p. cent }\end{array}$ & $\mathrm{C} / \mathrm{N}$ & $\mathrm{pH}$ & $\begin{array}{l}\mathrm{Ca} \\
(1)\end{array}$ & $\begin{array}{l}\mathbf{K} \\
(1)\end{array}$ & $\begin{array}{l}\mathrm{Mg} \\
(1)\end{array}$ & $\begin{array}{c}\mathrm{T} \\
(1)\end{array}$ & $\begin{array}{c}\mathrm{P}_{2} \mathrm{O}_{5} \\
\text { p. mille }\end{array}$ & $\begin{array}{l}\text { Fer } \\
\text { libre } \\
\text { Free } \\
\text { iron } \\
\text { p. cent }\end{array}$ & $\begin{array}{l}\text { A rgile } \\
\text { Clay } \\
\text { p. cent }\end{array}$ & $\begin{array}{c}\text { Limon } \\
\text { fin } \\
\text { Fine } \\
\text { silt } \\
\text { p. cent }\end{array}$ & $\begin{array}{c}\text { Limon } \\
\text { grossier } \\
\text { Coarse } \\
\text { silt } \\
\text { p. cent }\end{array}$ & $\begin{array}{c}\text { Sable } \\
\text { fin } \\
\text { Fine } \\
\text { sand } \\
\text { p. cent }\end{array}$ & $\begin{array}{c}\text { Sable } \\
\text { grossier } \\
\text { Coarse } \\
\text { sand } \\
\text { p. cent }\end{array}$ \\
\hline $\begin{array}{c}\mathrm{A}_{1} \\
\mathrm{~A}_{2} \mathrm{~g} \\
\mathrm{~A}_{2} \mathrm{~g} / \mathrm{Bg} \\
\mathrm{Bg}\end{array}$ & $\begin{array}{l}8,60 \\
2,33 \\
0,44 \\
0,27\end{array}$ & $\begin{array}{l}5,0 \\
1,36 \\
0,26 \\
0,16\end{array}$ & $\begin{array}{l}0,22 \\
0,09 \\
0,02 \\
0,02\end{array}$ & $\begin{array}{r}22,8 \\
15,3 \\
11,8 \\
7,3\end{array}$ & $\begin{array}{l}4,4 \\
5,5 \\
5,0 \\
5,1\end{array}$ & $\begin{array}{l}1,1 \\
0,5 \\
3,2 \\
4,5\end{array}$ & $\begin{array}{l}0,14 \\
0,05 \\
0,15 \\
0,19\end{array}$ & $\begin{array}{l}0,44 \\
0,29 \\
2,45 \\
3,76\end{array}$ & $\begin{array}{r}14,4 \\
7,2 \\
13,6 \\
17,8\end{array}$ & $\begin{array}{l}0,08 \\
0,21 \\
0,15 \\
0,19\end{array}$ & $\begin{array}{l}0,11 \\
0,18 \\
1,34 \\
1,39\end{array}$ & $\begin{array}{l}15,0 \\
11,6 \\
27,7 \\
34,5\end{array}$ & $\begin{array}{l}32,9 \\
43,7 \\
30,4 \\
28,5\end{array}$ & $\begin{array}{l}23,7 \\
25,3 \\
22,7 \\
18,8\end{array}$ & $\begin{array}{l}8,3 \\
6,5 \\
7,2 \\
6,5\end{array}$ & $\begin{array}{l}7,3 \\
8,0 \\
8,2 \\
7,2\end{array}$ \\
\hline
\end{tabular}


Le coefficient $K$ de perméabilité mesuré par la méthode du «trou de tarière » est égal à $1,9 \mathrm{~m} / \mathrm{j}$ en moyenne de la surface au " plancher » du pseudogley. Dans l'horizon $\mathrm{Bg}$, il est de $0,20 \mathrm{~m} / \mathrm{j}$.

La nappe perchée temporaire subit des fluctuations qui l'amènent à certaines périodes jusqu'en surface du sol; cette parcelle est réputée très " mouilleuse ».

Après exploitation du peuplement précédent (épicéas de première génération) le terrain a été envahi d'arbustes : tremble, bouleau, saule, bourdaine et, en ce qui concerne la végétation herbacée, de houlque molle, jonc, molinie, canche coespiteuse et de diverses espèces de carex. La présence de ces espèces ne peut nous étonner : certaines caractérisent le moder acidiphile, d'autres les sols à hydromorphie temporaire.

\subsection{2. Évaux.}

La pente du terrain est assez prononcée, surtout dans la partie « haute » de l'expérience (blocs 1 et 2) : environ $5 \mathrm{p}$. cent, puis $2 \mathrm{p}$. cent en moyenne pour les blocs 3 et 4 .

La roche-mère est formée des marnes du keuper recouvertes d'une mince couche de limons.

Le sol est un pélosol-pseudogley typique. L'horizon Alg a une texture limono-argileuse (tableau 2), une teneur moyenne en matière organique, de petites taches rouille d'hydromorphie remontant presque en surface, une structure grumeleuse. La teneur en matière organique diminue avec la profondeur; dans la partie inférieure de l'horizon, la surface couverte par les taches rouille augmente très nettement. La limite avec l'horizon suivant est tranchée.

$L$ 'horizon II Cg débute, suivant les endroits, entre -25 et $-30 \mathrm{~cm}$. Sa texture est fortement argileuse (63 p. cent d'argile), sa structure quelque peu prismatique, avec des slickensides (faces de glissement brillantes). Sa teinte est gris-verdâtre bariolé de rouille.

La roche-mère II $C$ débute à une profondeur variable, souvent voisine de -50 à $-60 \mathrm{~cm}$. Sa teinte est verdâtre, parfois mauve. Elle contient d'assez nombreux cailloux calcaires.

Le $p H$ de ce sol augmente avec la profondeur; il passe de 5,5 en Alg à 7,4 puis plus de 8 dans la roche-mère.

La nutrition en azote et cations est sans doute relativement satisfaisante; par contre, la teneur en phosphore paraît bien faible.

La végétation naturelle est tout à fait caractéristique d'un sol à hydromorphie temporaire très accentué, avec une très forte dominante de Molinia coerulea, mais aussi les espèces suivantes : Calamagrostis epigeios, Rhamnus frangula, Alnus glutinosa, Populus tremula, Carpinus betulus, etc. et, dans la partie la plus basse, des grands Carex.

En résumé, la différence essentielle du sol, en ce qui concerne l'hydromorphie, par rapport à celui d'Essegney, est la suivante : plancher de la nappe plus superficiel; d'autre part, la pente bien plus marquée laisse supposer un drainage latéral naturel nettement plus prononcé qu'à Essegney; par contre, le drainage profond est certainement bien moindre, en raison de la plus forte imperméabilité de la roche-mère (les marnes du Keuper sont très imperméables).

\section{2. - Les essences}

La surface disponible à Essegney nous permettait d'utiliser cinq essences qui ont été choisies suivant divers critères. Ce sont :

- I'Épicéa commun (Picea abies) essence couramment employée pour les reboisements des stations identiques à celle d'Essegney, en l'absence de connaissances précises sur le comportement d'autres espèces. Ses résultats y sont en général relativement bons, compte tenu des caractéristiques pédologiques défavorables ainsi que nous l'avons constaté dans une enquête (actuellement en cours) sur la production de l'Épicéa sur sols hydromorphes du Nord-Est.

Cependant son enracinement superficiel le rend sensible au chablis. D'autre part certains auteurs (SCHLENKER et al., 1969) le déconseillent vivement sur tout sol à hydromorphie temporaire en raison des dégradations quasi irréversibles du sol dont il serait la cause (de même d'ailleurs que d'autres espèces résineuses); mais cette opinion est loin d'être partagée par tous.

L'Épicéa a été retenu dans l'expérience d'Essegney d'une part comme critère vis-à-vis des quatre autres essences et aussi, bien sûr, afin de tester l'influence de l'assainissement sur sa propre croissance. 
TABLEAU $2-$ TABLE 2

Analyses du sol d'Évaux-et-Ménil

Analysis of Évaux-et-Ménil soil

\begin{tabular}{|c|c|c|c|c|c|c|c|c|c|c|c|c|c|c|c|}
\hline Hor. & $\begin{array}{c}\text { Mat. organique } \\
\text { Org. matter } \\
\text { p. cent }\end{array}$ & $\begin{array}{c}\mathrm{C} \\
\text { p. cent }\end{array}$ & $\begin{array}{l}\mathrm{N} \\
\text { p. cent }\end{array}$ & $\mathrm{C} / \mathrm{N}$ & $\mathrm{pH}$ & $\begin{array}{l}\mathrm{Ca} \\
\text { (1) }\end{array}$ & $\begin{array}{c}K \\
(1)\end{array}$ & $\begin{array}{l}\mathrm{Mg} \\
(1)\end{array}$ & $\begin{array}{c}\mathrm{T} \\
(1)\end{array}$ & $\begin{array}{c}\mathrm{P}_{2} \mathrm{O}_{5} \\
\text { p. mille }\end{array}$ & $\begin{array}{l}\text { Argile } \\
\text { Clay } \\
\text { p. cent }\end{array}$ & $\begin{array}{l}\text { Limon } \\
\text { fin } \\
\text { Fine } \\
\text { silt } \\
\text { p. cent }\end{array}$ & $\begin{array}{l}\text { Limon } \\
\text { grossier } \\
\text { Coarse } \\
\text { silt } \\
\text { p. cent }\end{array}$ & $\begin{array}{c}\text { Sable } \\
\text { fin } \\
\text { Fine } \\
\text { sand } \\
\text { p. cent }\end{array}$ & $\begin{array}{c}\text { Sable } \\
\text { grossier } \\
\text { Coarse } \\
\text { sand } \\
\text { p. cent }\end{array}$ \\
\hline $\begin{array}{c}\mathrm{A}_{1} \mathrm{~g} \\
\mathrm{IICg} \\
\text { IIC }\end{array}$ & $\begin{array}{l}5,2 \\
0,2 \\
0,1\end{array}$ & $\begin{array}{l}3,04 \\
0,14 \\
0,09\end{array}$ & 0,18 & 16,9 & $\begin{array}{l}5,5 \\
6,4 \\
7,4\end{array}$ & $\begin{array}{r}3,2 \\
8,3 \\
26,8\end{array}$ & $\begin{array}{l}0,38 \\
0,81 \\
0,84\end{array}$ & $\begin{array}{l}1,24 \\
2,50 \\
2,95\end{array}$ & $\begin{array}{l}12,0 \\
20,8 \\
28,8\end{array}$ & $\begin{array}{l}0,05 \\
0,02 \\
0,18\end{array}$ & $\begin{array}{l}23,1 \\
63,4 \\
66,8\end{array}$ & $\begin{array}{l}45,3 \\
21,9 \\
19,2\end{array}$ & $\begin{array}{r}20,1 \\
8,0 \\
7,3\end{array}$ & $\begin{array}{l}3,9 \\
1,0 \\
2,2\end{array}$ & $\begin{array}{l}2,4 \\
5,5 \\
4,4\end{array}$ \\
\hline
\end{tabular}

$?$

(1) m. é./100 g. 
Les plants utilisés, âgés de quatre ans $(2+2)$ proviennent de graines originaires de Pologne :

- le Douglas (Pseudotsuga menziesii).

La qualité de son bois et ses potentialités de production ne sont plus à vanter. Cependant, bien qu'assez plastique vis-à-vis du milieu, on pense généralement qu'une nappe relativement superficielle est une contre-indication à son emploi; mais il n'est pas impossible qu'un assainissement du sol ait un effet très bénéfique sur son comportement.

Les plants âgés de trois ans $(2+1)$ à la plantation proviennent de graines originaires de la chaîne des Cascades de l'état de Washington.

- le Mélèze d'Europe (Larix europaea D.C.).

Il présente un intérêt incontestable en raison de la rapidité initiale de sa croissance et des caractéristiques recherchées de son bois.

Les plants $(2+2)$ proviennent de graines originaires des Sudètes (les plants de cette provenance sont plus adaptés au climat lorrain que ceux des provenances alpines françaises).

- le Pin Weymouth (Pinus strobus L.).

Ses caractéristiques écologiques (très plastique, s'accomode en particulier de certains sols très hydromorphes) et technologiques (bois très recherché, surtout pour le déroulage) en font une essence théoriquement de grand intérêt. Mais son emploi est gravement hypothéqué par les ravages causés par la rouille vésiculeuse (Cronartium ribicola). Cependant cela n'interdit pas son usage, surtout dans des stations particulières défavorables et moyennant certaines conditions (peuplements peu étendus). Et il n'est pas interdit de penser que l'on pourra un jour combattre efficacement la rouille.

Les plants utilisés $(2+1)$ proviennent de graines originaires d'Autriche.

- le Cyprès de Lawson (Chamaecyparis lawsoniana Parlat).

La qualité de son bois est excellente et il serait peu sensible à l'hydromorphie.

Les plants $(2+2)$ proviennent de graines issues d'un reboisement en Haute-Loire.

Il est évident que d'autres essences mériteraient d'être testées; elles le seront à l'avenir dans toute la mesure du possible.

L'ensemble des essences choisies à Essegney présente l'avantage de ne pas nécessiter d'abri initial. Le maintien d'un tel abri créerait des conditions délicates pour l'expérience : difficulté d'exploitation ultérieure de l'abri sans créer de dommages à la plantation, introduction d'un facteur d'hétérogénéité écologique.

Cependant la plantation sous abri peut présenter des avantages certains en sol hydromorphe; elle limite en effet la remontée de la nappe qui est un des facteurs essentiels de la dégradation du sol. C'est pourquoi cette technique (et les essences qui s'y adaptent) pourrait être utilisée dans des essais futurs.

Seules les trois premières essences citées ont été utilisées à Évaux.

\section{3. - Modalités d'assainissement}

L'assainissement par drains enterrés, méthode la plus courante en agriculture, n'est pratiquement jamais utilisée en matière forestière en raison des risques de colmatage par les racines des arbres. C'est pourquoi nous n'avons pas utilisé, dans cette expérience tout au moins, ce type de drainage.

Quatre modalités d'assainissement ont été testées : billons et fossés à écartement d'environ $10 \mathrm{~m}$, $20 \mathrm{~m}$ et $40 \mathrm{~m}$.

On reconnaît en agriculture l'influence bénéfique d'un modelé du sol en planches bombées, ados ou billons, pour l'assainissement des pseudogleys à « plancher » relativement superficiel. Nous avons réalisé des ados de faible largeur en raison de la facilité de leur confection et de l'interprétation (tous les plants se trouvent dans les mêmes conditions, au sommet du billon).

Quant aux fossés, leur efficacité maxima est obtenue théoriquement si la nappe temporaire ne perturbe pas l'extension du système radiculaire des arbres en profondeur.

Or, les formules classiques de calcul des écartements de drainage (leur application ne donne ici que des résultats approximatifs, ne serait-ce que parce que la durée critique de submersion et sa 
périodicité admissible ne sont pas connues dans le cas de jeunes plants de résineux) montrent que même avec des fossés à écartement de $10 \mathrm{~m}$ nous sommes fort éloignés d'un optimum théorique.

Il est cependant hors de question, en matière forestière, sauf cas très particuliers, de confectionner des fossés distants de moins de $10 \mathrm{~m}$, et même cette dernière distance ne pourrait être matériellement envisageable dans la plupart des cas.

Par ailleurs il est vraisemblable que des fossés distants de plus de $40 \mathrm{~m}$ n'auraient guère d'influence sur le niveau de la nappe, donc sur le comportement des plants.

C'est pourquoi nous avons choisi de tester des écartements d'environ 10, 20 et $40 \mathrm{~m}$.

A Évaux, en raison du caractère superficiel de la nappe perchée, il est probable, d'une part qu'un même écartement de fossés aura un effet plus réduit sur le rabattement de la nappe qu'à Essegney, d'autre part que le mode d'assainissement le plus efficace sera constitué par des billons. Cependant, afin que la comparaison puisse être plus complète nous avons conservé à Évaux les mêmes modalités qu'à Essegney.

\section{4. - Le dispositif expérimental}

Il s'agit d'expériences en blocs. La figure 1 représente, à titre d'exemple, le plan de l'expérience d'Essegney.

En raison des déblais, les plants les plus proches des fossés sont situés seulement à deux mètres de leur bord. Pour cette raison et afin de conserver le même nombre de plants par ligne, quelle que soit la modalité « fossés », les distances entre axe de deux fossés successifs sont en réalité de 39,5 21,5 et $12,5 \mathrm{~m}$ et non de 40, 20 et 10 mètres. Par commodité, nous continuerons cependant à désigner les diverses modalités par ces derniers chiffres.

Les billons sont distants de 2,30 m l'un de l'autre; ils sont au nombre de 16 à Essegney, de 20 à Évaux. Les plants disposés sur les sommets de billons forment à Essegney et Évaux respectivement 6 et 5 lignes successives distantes de 1,50 m l'une de l'autre dans chaque parcelle. La longueur de terrain occupée par chaque parcelle étant, à Essegney supérieure à celle qui correspond à une parcelle à modalité fossés, seules quatre essences y ont été plantées sur billons : le Pin Weymouth n'a pu y être testé.

\section{5. - Réalisation}

Un labour croisé a été réalisé à l'aide d'une charrue de deux tonnes de type Rome TMR 6/30 tractée par un bulldozer D4 Caterpillar et munie de 6 disques crênelés.

Les fossés ont été creusés au moyen d'une pelle mécanique sur chenilles de type Yumbo Y 45 munie d'un godet trapézoïdal. A Essegney leur profondeur est d'environ $0,60 \mathrm{~m}$ en amont et $0,80 \mathrm{~m}$ en aval, leur largeur au plafond est voisine de $0,45 \mathrm{~m}$, leur ouverture de l'ordre de $1 \mathrm{~m}$. A Évaux, leur profondeur est de 0,50 à $0,60 \mathrm{~m}$, leur ouverture de $0,90 \mathrm{~m}$.

Les billons ont été réalisés par un double passage d'une charrue à disque de type Rome (même charrue que pour le labour, mais « fermée », c'est-à-dire l'axe des deux séries de disques formant l'angle maximum). Leur dénivellation (de creux à sommet) est de l'ordre de $0,50 \mathrm{~m}$. Des rigoles prolongent le creux des billons jusqu'au collecteur suffisamment éloigné de la zone expérimentale. A Essegney, en raison de la très faible pente du terrain, l'eau stagne partiellement dans le creux des billons des blocs 3 et 4 .

A Évaux, l'axe des fossés et billons est incliné par rapport à la ligne de plus grande pente, mais sans doute moins qu'il n'aurait été souhaitable; mais pour des raisons matérielles (conformation de la parcelle) il n'a pas été possible de faire en sorte que l'angle entre l'axe des fossés et les courbes de niveau soit plus faible.

Seule J'eau des précipitations atteignant la parcelle même est à évacuer en raison de la présence de fossés de ceinture.

La plantation a été effectuée en potets de $0,30 \mathrm{~m}$ de rayon et $0,20 \mathrm{~m}$ de profondeur, confectionnés et fertilisés plusieurs mois auparavant : par potet, $30 \mathrm{~g}$ de sulfate de potassium dans les deux expériences, $100 \mathrm{~g}$ d'hyperphosphate Reno à Essegney, $60 \mathrm{~g}$ de superphosphate triple à Évaux (réaction moins acide du sol). Au printemps 1970, on a épandu au pied de chaque plant $10 \mathrm{~g}$ d'ammonitrate à $34 \mathrm{p}$. cent à Essegney, $16 \mathrm{~g}$ de sulfate d'ammonium à Évaux.

Les travaux du sol ont été réalisés au cours de l'été 1968, la plantation début avril 1969. 

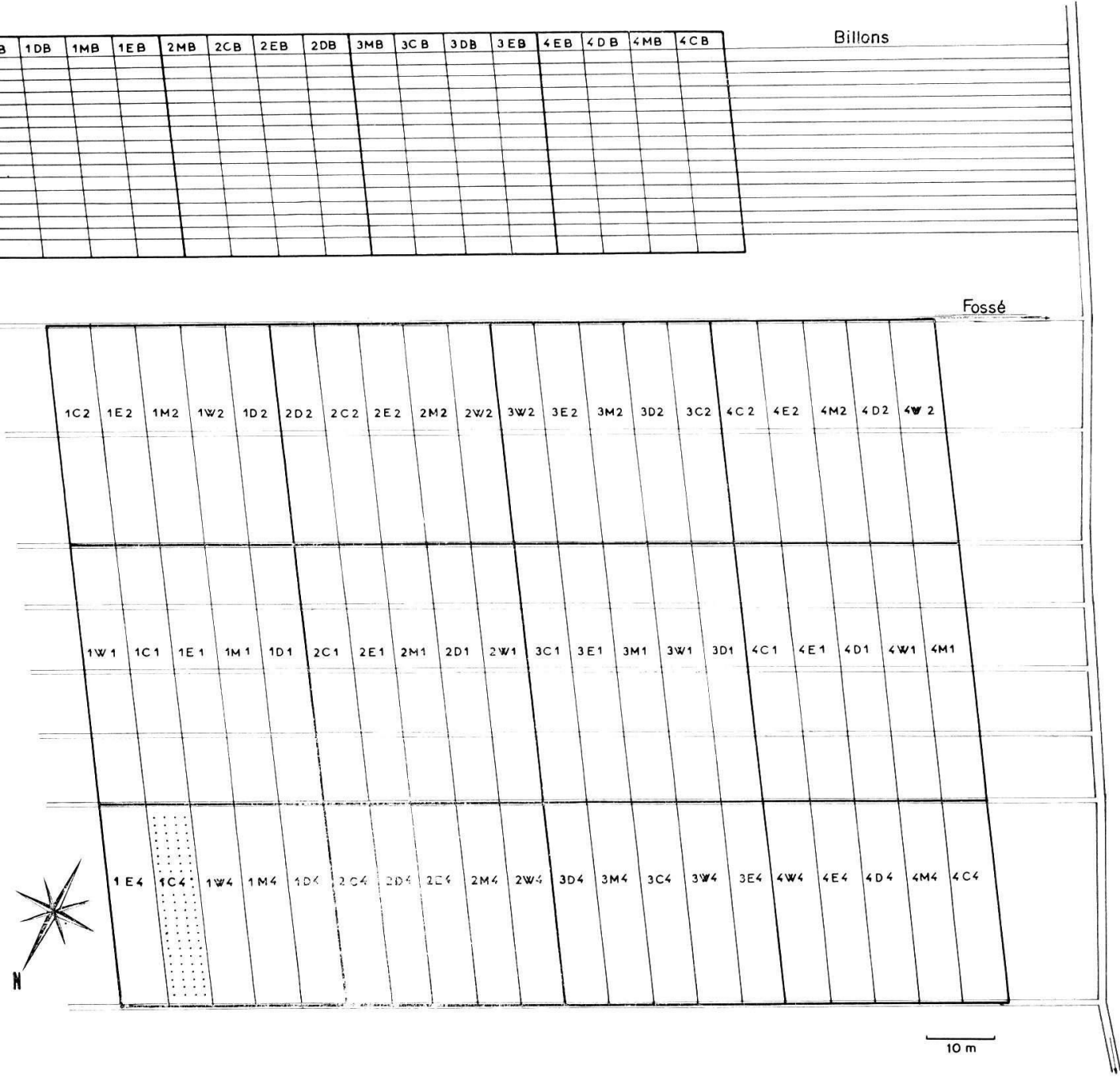

FIG. 1. - Plan de l'expérience d'Essegney. Légende : pour chaque parcelle, dans l'ordre :

- $n^{\circ}$ du bloc,

- lettre initiale du nom de genre,

- modalité d'assainissement, $1=$ fossés distants de $10 \mathrm{~m}$, $2=$ fossés distants de $20 \mathrm{~m}$, $4=$ fossés distants de $40 \mathrm{~m}$, $\mathrm{B}=$ billons.
FIG. 1. - Scheme of Essegney experiment

For each plot, successively :

- number of the block,

- first letter of genous noun,

- modality of drainage,

$1=$ ditches distant of $10 \mathrm{~m}$,

$2=$ ditches distant of $20 \mathrm{~m}$,

$4=$ ditches distant of $40 \mathrm{~m}$,

$\mathrm{B}=$ ridges.

Des piézomètres ont été installés, les uns à mi-distance entre deux fossés consécutifs ainsi que sur des sommets de billons, les autres (distants de $1,50 \mathrm{~m}$ ) suivant deux lignes perpendiculaires aux fossés sur toute la largeur de l'expérience. Cependant, en ce qui concerne cette deuxième série de piézomètres, nous n'avons jusqu'à présent effectué de relevés que sur l'une des lignes, à Essegney. 


\section{6. - Interprétation}

Les déterminations essentielles concernent le taux de reprise des plants ainsi que les pousses annuelles. Les moyennes des pousses seront comparées par analyse de variance à deux facteurs contrôlés (bloc et traitement).

Il est évident que, dans un premier temps tout au moins, il y aura pratiquement 5 expériences séparées : en effet il ne saurait être question de comparer quantitativement le comportement d'essences à rythme de croissance différent. Cependant cette comparaison pourra s'avérer fort instructive au bout d'un certain nombre d'années.

On pourra contester l'intérêt d'avoir fertilisé les potets; en effet il y a peut-être interaction assainissement-engrais, l'effet relatif d'un assainissement correct par rapport à un témoin peu drainé étant peut-être différent en l'absence de fertilisation. Cependant il nous a paru qu'en apportant des éléments fertilisants, nous étudierions de façon plus correcte le facteur assainissement par suppression d'un autre facteur limitant éventuel; et de cette façon, lors de comparaisons des résultats entre expériences du même type, on pourra penser valablement que les différences constatées ont pour origine principale des conditions d'hydromorphie différentes. L'idéal aurait d'ailleurs consisté à «spliter » chaque parcelle en deux sous-parcelles, l'une fertilisée et l'autre non. Le manque de superficie suffisante nous l'a interdit.

\section{III. - RÉSULTATS}

\section{1. - Relèves piézométriques}

Des relevés ont été effectués à plusieurs reprise au cours des périodes d'engorgement du sol.

\subsection{Essegney.}

Les plus intéressants parmi ces relevés concernent la ligne piézométrique traversant l'expérience dans toute sa largeur (à part les billons) et s'étendant à une portion de terrain non assaini (témoin). Les courbes correspondant à trois d'entre eux sont reproduites sur la figure 2; elles ont été obtenues en reliant d'un trait les points représentatifs du niveau de l'eau dans les piézomètres successifs. On peut faire les constatations suivantes :

- A une même date, il y a souvent une différence assez sensible de niveau entre deux piézomètres successifs, ce qui donne aux courbes un aspect en lignes fortement brisées. La cause est probablement constituée par les irrégularités de la surface du sol ainsi qu'une certaine hétérogénéité horizontale de ses propriétés. Ces différences se retrouvent identiques, du moins grossièrement, aux différentes dates de mesure.

- Néanmoins, du moins pour les modalités 40 et $20 \mathrm{~m}$, on constate qu'une courbe continue moyenne aurait une forme convexe assez proche de la courbe théorique.

- Les épaisseurs de sol assaini par rapport à la modalité $40 \mathrm{~m}$ considérée comme témoin relatif pour les trois dates considérées sont représentées au tableau 3. Ces épaisseurs sont très voisines aux trois dates de mesures, c'est-à-dire pour différents niveaux d'affleurement de la nappe. L'effet supplémentaire du drainage est nettement plus marqué pour la modalité $20 \mathrm{~m}$ (par rapport aux $40 \mathrm{~m}$ ) que pour la modalité $10 \mathrm{~m}$ (par rapport aux $20 \mathrm{~m}$ ), surtout si l'on considère les épaisseurs moyennes. D'autre part, le sommet des billons présente une 


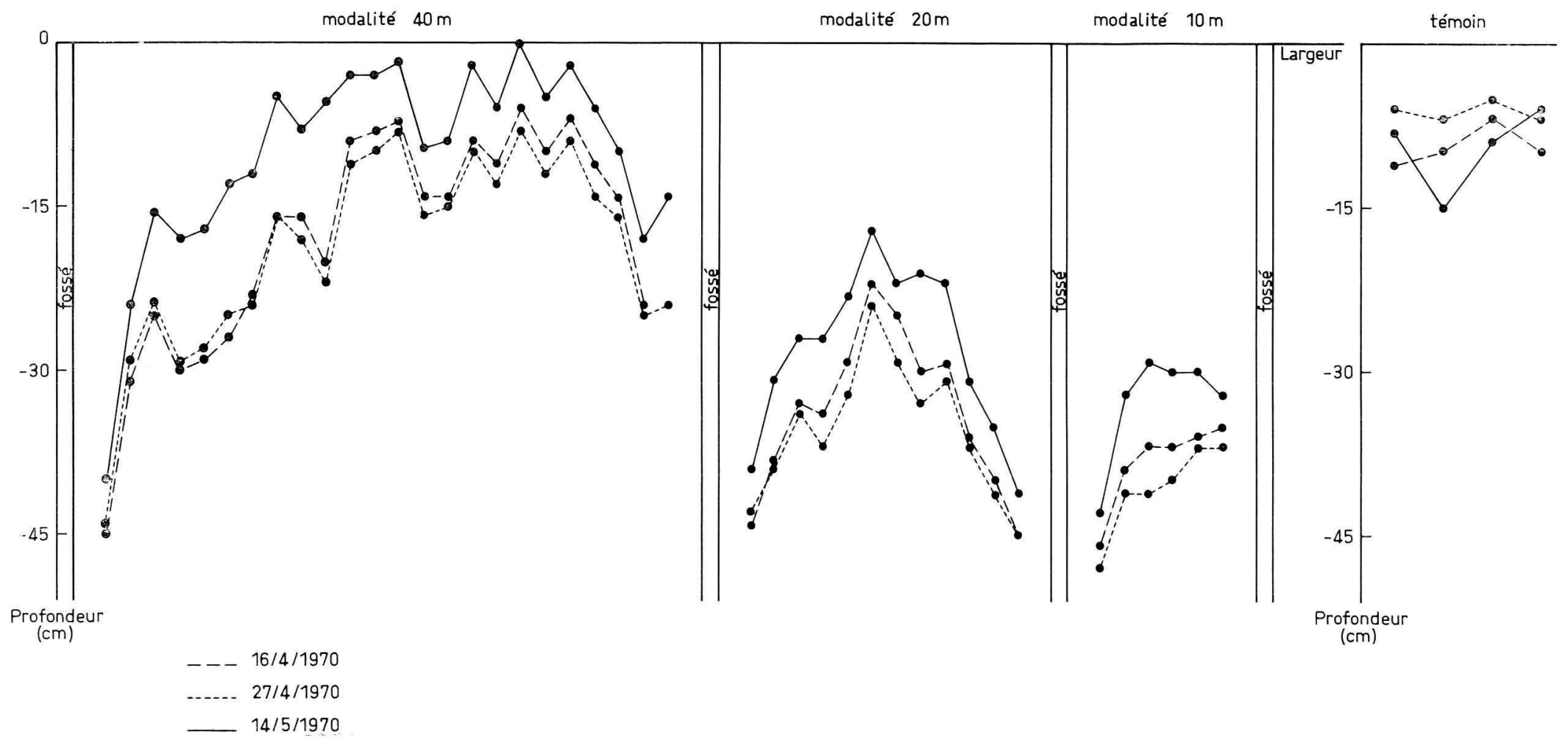

FIG. 2. - Fluctuations de la nappe sur une ligne perpendiculaire aux fossés (chaque courbe des modalités 20 et $10 \mathrm{~m}$ est la moyenne respectivement de 2 et 4 courbes).

FIG. 2. - Fluctuations of the water-table along a line perpendicular to the ditches (every curve for the modalities 20 and $10 \mathrm{~m}$. is the average respectively of 2 and 4curves). 
Épaisseur du sol assaini par rapport à la modalité « $40 \mathrm{~m} \gg$ à Essegney

Thickness of soil dried comparatively to the modality « $40 \mathrm{~m}$ 》 at Essegney

\begin{tabular}{|c|c|c|c|c|c|c|c|}
\hline \multirow[b]{2}{*}{$\begin{array}{l}\text { Modalité } \\
\text { Modality }\end{array}$} & \multicolumn{3}{|c|}{$\begin{array}{c}\text { Épaisseur au point } \\
\text { où la nappe est la plus superficielle (1) } \\
\text { Thickness at the point where } \\
\text { the water-table is the most shallow } \\
(\mathrm{cm})\end{array}$} & \multicolumn{4}{|c|}{$\begin{array}{c}\text { Épaisseur moyenne } \\
\text { Mean thickness } \\
(\mathrm{cm})\end{array}$} \\
\hline & $40 \mathrm{~m}$ & $20 \mathrm{~m}$ & $10 \mathrm{~m}$ & $40 \mathrm{~m}$ & $20 \mathrm{~m}$ & $10 \mathrm{~m}$ & $\begin{array}{c}\text { billons } \\
\text { ridges }\end{array}$ \\
\hline $\begin{array}{l}16 / 4 / 70 \\
27 / 4 / 70 \\
14 / 5 / 70\end{array}$ & $\begin{array}{l}0 \\
0 \\
0\end{array}$ & $\begin{array}{l}14,5 \\
15 \\
15,5\end{array}$ & $\begin{array}{l}25 \\
26 \\
23,5\end{array}$ & $\begin{array}{l}0 \\
0 \\
0\end{array}$ & $\begin{array}{l}16 \\
16 \\
18\end{array}$ & $\begin{array}{l}20 \\
22 \\
23\end{array}$ & $\begin{array}{l}8,5 \\
6\end{array}$ \\
\hline
\end{tabular}

(1) Il s'agit, pour 20 et $10 \mathrm{~m}$, des moyennes concernant les points les plus superficiels de la nappe respectivement des 2 et 4 courbes (il y a 2 courbes pour les $20 \mathrm{~m}$, car 3 fossés, et 4 courbes pour les $10 \mathrm{~m}$, car 5 fossés), quelle que soit leur position par rapport aux fossés.

That is, for 20 and $10 \mathrm{~m}$, the means concerning the most shallow points of the water-table respectively of the 2 and 4 curves (there are 2 curves for the $20 \mathrm{~m}$, because 3 ditches, and 4 curves for the $10 \mathrm{~m}$, because 5 ditches), whatever their position in respect to the ditches may be.

épaisseur de sol sain nettement inférieure à celle de la modalité $20 \mathrm{~m}$, même si l'on ne considère que les blocs 1 et 2 .

Très souvent, le niveau de la nappe dans le piézomètre le plus proche d'un fossé est très nettement inférieur à son niveau dans tous les autres piézomètres.

Il semble que le niveau de la nappe dans une zone médiane (éloignée des deux fossés) de la modalité $40 \mathrm{~m}$, soit très proche du niveau de la nappe sans drainage. Cette zone pourra donc sans grande erreur être considérée comme une zone témoin non assainie. L'ensemble de la modalité $40 \mathrm{~m}$ pourra d'ailleurs être utilisée très valablement comme témoin relatif par rapport aux autres modalités, en raison des très sensibles différences de nappe constatées.

Les remarques précédentes justifient les trois écartements de fossés testés dans l'expérience.

\subsection{2. Évaux.}

A titre d'exemple, nous reproduisons dans le tableau 4 pour deux dates différentes l'épaisseur de sol assaini à mi-distance entre deux fossés consécutifs et au sommet des billons. Pour la première de ces deux dates, nous indiquons les moyennes des relevés effectués à Essegney dans des piézomètres dont la position correspond à celle des piézomètres d'Évaux.

A ces deux dates, la nappe affleure au centre de la modalité $40 \mathrm{~m}$ respectivement à 10 et $16 \mathrm{~cm}$ de la surface du sol à Évaux et à $14 \mathrm{~cm}$ à Essegney.

Après avoir insisté sur le fait que ces chiffres représentent une épaisseur de sol assaini moyenne pour les billons, et en principe minima pour les modalités fossés, donc que les 


\section{TABLEAU 4 - TABLE 4}

Épaisseur du sol assaini, par rapport à la modalité « $40 \mathrm{~m}$ », au centre des modalités « fossés » et au sommet des billons (moyennes de plusieurs mesures, en $\mathrm{cm}$ )

Thickness of soil dried comparatively to the modality « $40 \mathrm{~m}$ », in the center of the modalities ditches and on the top of the ridges (means of several measures, in $\mathrm{cm}$ )

\begin{tabular}{ll|c|c|c|c}
\hline & & $40 \mathrm{~m}$ & $20 \mathrm{~m}$ & $10 \mathrm{~m}$ & $\begin{array}{r}\text { billons } \\
\text { ridges }\end{array}$ \\
\cline { 3 - 5 } & & & & & \\
$28 / 1 / 70$ & Évaux & Essegney & 0 & 2 & 4 \\
$16 / 4 / 70$ & Évaux & 0 & 6 & 9 & 15 \\
& & 0 & 1 & 1 & 13 \\
\hline
\end{tabular}

chiffres concernant fossés et billons ne peuvent être comparés d'une manière absolue, on peut faire les constatations suivantes :

- le 28 janvier, bien que l'ordre logique soit respecté, l'épaisseur de sol assaini à Évaux au centre des modalités 10 et $20 \mathrm{~m}$ par rapport aux « $40 \mathrm{~m}$ » est très faible. Il existe une différence très sensible entre ces chiffres et ceux relevés au sommet des billons. A cette même date l'épaisseur de sol assaini dans les modalités 20 et $10 \mathrm{~m}$ est plus marquée à Essegney; par contre, l'épaisseur de sol assaini au sommet des billons n'y est pas plus importante qu'au centre des $10 \mathrm{~m}$, au contraire.

- le 16 avril, le sol n'est pratiquement pas assaini à Évaux au centre des modalités 20 et $10 \mathrm{~m}$, toujours par rapport aux $40 \mathrm{~m}$, mais il l'est très nettement au sommet des billons.

- Tout cela nous permet de supposer que les billons seront bien plus efficaces, proportionnellement, à Évaux qu'à Essegney. De plus, le drainage par fossés ne semble guère efficace à Évaux. Dès lors, on peut penser que c'est sur les billons que les résultats les plus intéressants seront enregistrés (en ce qui concerne les plants).

- Il s'ensuit également que le comportement des plants à proximité du centre de la modalité $40 \mathrm{~m}$ pourra être considéré comme peu différent de celui d'une modalité témoin sans assainissement.

Il est cependant nécessaire d'être prudent dans nos conclusions; des comparaisons plus valables de niveaux de nappes entre les différentes modalités à Évaux, et entre Essegney et Évaux ne pourront être effectuées que sur des moyennes de niveau de nappe pour toute la largeur de chaque modalité, et non pas seulement sur les niveaux au centre de chaque modalité. Ces moyennes pourront être prochainement calculées à partir de relevés piézométriques effectués sur les deux lignes de piézomètres traversant toute l'expérience perpendiculairement aux fossés.

\section{2. - Résultats de l'assainissement}

\subsection{Mortalité initiale.}

Les données recueillies fin 1970 sont consignées dans les tableaux 5 et 6 , le nombre total de plants étant au départ, par essence et par modalité, de 384 dans chacune des deux expériences. 
TABleau 5 - TABle 5

Mortalité au cours des deux premières années à Essegney

Mortality during the first two years at Essegney

\begin{tabular}{|c|c|c|c|c|c|c|c|c|c|c|}
\hline \multirow{2}{*}{$\begin{array}{l}\text { Espèces } \\
\text { Species }\end{array}$} & \multicolumn{2}{|l|}{$10 \mathrm{~m}$} & \multicolumn{2}{|l|}{$20 \mathrm{~m}$} & \multicolumn{2}{|l|}{$40 \mathrm{~m}$} & \multicolumn{2}{|l|}{ billons } & \multirow{2}{*}{$\begin{array}{c}\text { Test du } \mathrm{X}^{2} \\
\text { entre les } 3 \\
\text { modalités fossés } \\
\text { Test of } \mathrm{X}^{2} \\
\text { between the } 3 \\
\text { modalities } \\
\text { « ditches » }\end{array}$} & \multirow{2}{*}{$\begin{array}{c}\text { Différences } \\
\text { significatives } \\
2 \text { à } 2 \\
\text { two by two } \\
\text { significant } \\
\text { differences }\end{array}$} \\
\hline & $\begin{array}{c}\text { Nombre } \\
\text { de morts } \\
\text { Quantity } \\
\text { of dead plants }\end{array}$ & $\begin{array}{c}\text { P. } \\
\text { cent }\end{array}$ & $\begin{array}{c}\text { Nombre } \\
\text { de morts } \\
\text { Quantity } \\
\text { of dead plants }\end{array}$ & $\begin{array}{c}\mathrm{p} . \\
\text { cen }\end{array}$ & $\begin{array}{c}\text { Nombre } \\
\text { de morts } \\
\text { Quantity } \\
\text { of dead plants }\end{array}$ & $\begin{array}{c}\text { p. } \\
\text { cent }\end{array}$ & $\begin{array}{c}\text { Nombre } \\
\text { de morts } \\
\text { Quantity } \\
\text { of dead plants }\end{array}$ & $\begin{array}{c}\text { p. } \\
\text { cent }\end{array}$ & & \\
\hline $\begin{array}{l}\text { Épicéa } \ldots \ldots \ldots \ldots \\
\text { Norway Spruce }\end{array}$ & 17 & 4 & 11 & 3 & 14 & 4 & 54 & 14 & NS & \\
\hline $\begin{array}{l}\text { Douglas } \ldots \ldots \ldots \ldots \\
\text { Douglas fir }\end{array}$ & 15 & 4 & 25 & 7 & 69 & 18 & 72 & 19 & TS (1 p. mille) & $\begin{array}{r}10 \mathrm{~m}-40 \mathrm{~m} \\
\text { et } 20 \mathrm{~m}-40 \mathrm{~m}\end{array}$ \\
\hline $\begin{array}{l}\text { Mélèze .............. } \\
\text { European Larch }\end{array}$ & 28 & 7 & 21 & 5 & 45 & 12 & 75 & 20 & TS (1 p. mille) & $\begin{array}{r}10 \mathrm{~m}-40 \mathrm{~m} \\
\text { et } 20 \mathrm{~m}-40 \mathrm{~m}\end{array}$ \\
\hline $\begin{array}{l}\text { Weymouth } . . . \ldots \ldots \\
\text { White pine }\end{array}$ & 11 & 3 & 15 & 4 & 21 & 5 & & & NS & \\
\hline $\begin{array}{l}\text { Cyprès de Lawson } \\
\text { Lawson's Cypress }\end{array}$ & 106 & 28 & 152 & 40 & 123 & 32 & 43 & 11 & S (1 p. cent & $\begin{array}{r}10 \mathrm{~m}-20 \mathrm{~m} \\
\text { et } 40 \mathrm{~m}-20 \mathrm{~m}\end{array}$ \\
\hline
\end{tabular}




\section{TABLEAU 6 - TABLE 6}

Mortalité au cours des deux premières années à Évaux-et-Ménil

Mortality during the first two years, at Évaux-et-Ménil

\begin{tabular}{|c|c|c|c|c|c|c|c|c|c|c|}
\hline \multirow{2}{*}{$\begin{array}{l}\text { Espèces } \\
\text { Species }\end{array}$} & \multicolumn{2}{|l|}{$10 \mathrm{~m}$} & \multicolumn{2}{|l|}{$20 \mathrm{~m}$} & \multicolumn{2}{|l|}{$40 \mathrm{~m}$} & \multicolumn{2}{|l|}{$\begin{array}{l}\text { Billons } \\
\text { Ridges }\end{array}$} & \multirow{2}{*}{$\begin{array}{c}\text { Test du X } \\
\text { entre les } \\
4 \text { modalités } \\
\text { Test of } X^{1} \\
\text { between the } 4 \\
\text { modalities }\end{array}$} & \multirow{2}{*}{$\begin{array}{c}\text { Différences } \\
\text { significatives } \\
2 \text { à } 2 \\
\text { two by two } \\
\text { significant } \\
\text { differences }\end{array}$} \\
\hline & $\begin{array}{c}\text { Nombre } \\
\text { de morts } \\
\text { Quantity } \\
\text { of dead plants }\end{array}$ & $\begin{array}{c}\text { p. } \\
\text { cent }\end{array}$ & $\begin{array}{c}\text { Nombre } \\
\text { de morts } \\
\text { Quantity } \\
\text { of dead plants }\end{array}$ & $\begin{array}{c}\text { p. } \\
\text { cent }\end{array}$ & $\begin{array}{c}\text { Nombre } \\
\text { de morts } \\
\text { Quantity } \\
\text { of dead plants }\end{array}$ & $\begin{array}{c}\mathrm{p} . \\
\text { cent }\end{array}$ & $\begin{array}{c}\text { Nombre } \\
\text { de morts } \\
\text { Quantity } \\
\text { of dead plants }\end{array}$ & $\begin{array}{c}\text { p. } \\
\text { cent }\end{array}$ & & \\
\hline $\begin{array}{l}\text { Épicéa } \ldots \ldots \\
\text { Spruce }\end{array}$ & 13 & 3 & 22 & 6 & 18 & 5 & 15 & 4 & NS & \\
\hline $\begin{array}{l}\text { Douglas } \ldots \ldots \ldots \ldots \\
\text { Douglas fir }\end{array}$ & 86 & 23 & 112 & 29 & 153 & 40 & 24 & 6 & TS (1 p. mille) & Toutes \\
\hline $\begin{array}{l}\text { Mélèze } \ldots \ldots \ldots \ldots \\
\text { Larch }\end{array}$ & 38 & 10 & 38 & 10 & 45 & 12 & 30 & 8 & NS & \\
\hline
\end{tabular}


On considérera conventionnellement comme faible un taux de mortalité $<8 \mathrm{p}$. cent et comme élevé un taux $>20$ p. cent.

\subsection{Essegney.}

Compte rendu que le Pin Weymouth n'a pas été testé sur billons, on peut effectuer les constatations suivantes :

- la mortalité est faible donc le taux de reprise satisfaisant pour l'Épicéa et le Weymouth dans les modalités 10,20 et 40 m et pour le Douglas et le Mélèze dans les modalités 10 et $20 \mathrm{~m}$.

- la mortalité est moyenne pour toutes les essences sur billons ainsi que pour le Douglas et le Mélèze dans la modalité $40 \mathrm{~m}$.

- la mortalité est forte pour le Cyprès de Lawson dans toutes les modalités « fossés ». Cependant le fait que sa mortalité soit la plus élevée dans la modalité $20 \mathrm{~m}$ est assez incompréhensible.

- les résultats concernant les billons sont a priori surprenants : la mortalité y est supérieure pour trois essences sur 4 à ce qu'elle est dans la modalité $40 \mathrm{~m}$, c'est-à-dire sur un sol probablement très peu assaini. La cause en est vraisemblablement dans le fait signalé précédemment que l'eau stagne souvent dans les creux de billons en raison de la très faible pente du terrain principalement dans les blocs 3 et 4 . En fait, si l'on ne considère que les blocs 1 et 2, la mortalité sur billons pour les trois essences est bien moins élevée, en général intermédiaire entre celles des modalités 20 et $40 \mathrm{~m}$ pour une essence donnée.

- En ce qui concerne le Cyprès de Lawson, les résultats sont tout à fait différents de ceux des autres essences; c'est sur billons que son taux de reprise est très nettement le plus élevé.

- En conclusion, on peut affirmer que jusqu'à la fin de la $2^{\mathrm{e}}$ année de plantation, le drainage n'a pas amélioré le taux de reprise (qui est bon de toutes façons) de l'Épicéa et du Weymouth, alors qu'un drainage à écartement de fossés de $20 \mathrm{~m}$ améliore assez nettement celui du Douglas et du Mélèze et un billonnage celui du Cyprès de Lawson.

\subsection{Evaux.}

On constate que :

- la mortalité est faible :

- pour l'Épicéa dans toutes les modalités,

- pour le Douglas sur billons.

- elle est moyenne pour le Mélèze dans toutes les modalités,

- elle est élevée pour le Douglas dans les trois modalités " fossés», principalement dans les $40 \mathrm{~m}$, à tel point qu'il ne saurait pratiquement être question d'utiliser le Douglas, dans un sol de ce type, sans effectuer de travaux d'assainissement.

- la plantation sur billons améliore considérablement le taux de reprise du Douglas (mortalité passant de $40 \mathrm{p}$. cent à $6 \mathrm{p}$. cent) et un peu celui du Mélèze. 
- le drainage par fossés à écartement de 10 ou $20 \mathrm{~m}$ n'améliore le taux de reprise par rapport au témoin relatif $(40 \mathrm{~m})$ que dans le cas du Douglas, et d'une façon bien moindre que le drainage par billons.

\subsection{La croissance en hauteur.}

Les mesures des pousses annuelles ont été effectuées fin 1969 et fin 1970. Les différentes modalités ont été comparées par analyse de variance puis éventuellement comparaison des moyennes 2 à 2 .

La tendance générale indiquée par ces calculs est identique, pour chaque essence, pour les pousses des deux années, les différences étant généralement plus sensibles en 1970 qu'en 1969 (en raison sans doute de la plus forte croissance générale).

C'est pourquoi nous nous bornerons à présenter les résultats obtenus pour la somme des deux pousses, c'est-à-dire pour la croissance pendant les deux premières années de plantation.

3.221. Essegney (tableau 7).

TABLEAU 7 - TABLE 7

Longueur des pousses $1969+1970$ à Essegney (en cm)

Length of the shoots $1969+1970$ at Essegney (in cm)

\begin{tabular}{|c|c|c|c|c|c|c|c|c|}
\hline $\begin{array}{l}\text { Espèces } \\
\text { Species }\end{array}$ & $10 \mathrm{~m}$ & $20 \mathrm{~m}$ & $40 \mathrm{~m}$ & $\begin{array}{l}\text { Billons } \\
\text { Ridges }\end{array}$ & $\begin{array}{c}\text { F théorique } \\
\text { à } 5 \text { et } 1 \text { p. cent } \\
\text { Theorical F } \\
\text { at } 5 \text { and } 1 \text { p. cent }\end{array}$ & $\begin{array}{c}\text { F calculé } \\
\text { calculated F }\end{array}$ & $\begin{array}{l}\text { P.P.D.S. } \\
\text { à } 5 \text { p. cent } \\
\text { L.S.D. } \\
\text { at } 5 \text { p. cent }\end{array}$ & $\begin{array}{l}\text { P.P.D.S. } \\
\text { à } 1 \text { p. cent } \\
\text { L.S.D. } \\
\text { at } 1 \text { p. cent }\end{array}$ \\
\hline $\begin{array}{l}\text { Épicéa ..... } \\
\text { Spruce }\end{array}$ & 21,6 & 21,1 & 16,2 & 18,9 & $3,86-6,99$ & $10,01^{++}$ & 2,5 & 3,6 \\
\hline $\begin{array}{l}\text { Douglas.... } \\
\text { Douglas fir }\end{array}$ & 43,1 & 43,8 & 29,6 & 35,0 & $3,86-6,99$ & $8,75^{++}$ & 6,1 & 8,8 \\
\hline $\begin{array}{l}\text { Mélèze } . . . \\
\text { Larch }\end{array}$ & 56,2 & 48,6 & 34,4 & 44,5 & $3,86-6,99$ & $8,00^{++}$ & 9,9 & 14,3 \\
\hline $\begin{array}{l}\text { Weymouth } \\
\text { White pine }\end{array}$ & 33,8 & 32,8 & 32,1 & & $5,14-10,92$ & 1,87 & & \\
\hline $\begin{array}{l}\text { Cyprès de } \\
\text { Lawson } \cdots \\
\text { Lawson's } \\
\text { Cypress }\end{array}$ & 31,2 & 26,4 & 24,1 & 38,6 & $3,86-6,99$ & 3,76 & & \\
\hline $\begin{array}{l}\text { Cyprès de } \\
\text { Lawson.... } \\
\text { (blocs } 1 \text { et } 2 \\
\text { seulement) } \\
\text { Lawson's } \\
\text { Cypress } \\
\text { (only blocks } \\
1 \text { and 2) }\end{array}$ & 31,1 & 30,6 & 23,0 & 47,8 & $9,28-29,46$ & $55,99^{++}$ & 6,3 & 11,5 \\
\hline
\end{tabular}




\section{Épicéa.}

L'augmentation de croissance en hauteur provoquée par un drainage à écartement de $10 \mathrm{~m}$ par rapport à l'écartement $40 \mathrm{~m}$ est de $33 \mathrm{p}$. cent.

Par rapport à la croissance des plants situés à proximité du centre de la modalité $40 \mathrm{~m}$ (témoins supposés " absolus 》) (moyenne des pousses : 14,9 cm), cette augmentation est de $45 \mathrm{p}$. cent. Précisons cependant que nous n'attachons pas de valeur fondamentale à ce dernier chiffre : il n'est fourni qu'à titre indicatif, car on considère comme témoin absolu un nombre de plants bien inférieur à celui des modalités complètes (les 4 plants centraux de chaque ligne, dans la modalité $40 \mathrm{~m}$, donc 64 plants en tout). Cette remarque sera évidemment valable également pour les autres essences ainsi qu’à Évaux-et-Ménil.

\section{Douglas.}

L'augmentation de croissance en hauteur provoquée par un drainage à écartement $20 \mathrm{~m}$ par rapport au témoin relatif (écartement $40 \mathrm{~m}$ ) est de $48 \mathrm{p}$. cent.

Par rapport au témoin « absolu » (moyenne des pousses : 23,7 cm) cette augmentation est de 84 p. cent.

\section{Mélèze.}

L'augmentation de croissance en hauteur provoquée par un drainage à écartement $10 \mathrm{~m}$ par rapport au témoin relatif (écartement $40 \mathrm{~m}$ ) est de $63 \mathrm{p}$. cent et par rapport au témoin «absolu » (centre de la modalité $40 \mathrm{~m}$, moyenne de pousses $32 \mathrm{~cm}$ ) de $76 \mathrm{p}$. cent.

\section{Pin Weymouth.}

Le drainage n’a pas jusqu'à présent amélioré sa croissance en hauteur de manière significative.

\section{Cyprès de Lawson.}

L'essai n'est pas significatif. Cependant, comme $F$ calculé est très proche du F théorique à $5 \mathrm{p}$. cent $(3,76$ et 3,86$)$ nous avons tout de même effectué une comparaison des moyennes de pousses 2 à 2 .

Les différences suivantes sont significatives à $5 \mathrm{p}$. cent :

$$
\begin{aligned}
& \text { billons }-40 \mathrm{~m} \\
& \text { billons }-20 \mathrm{~m}
\end{aligned}
$$

Ce sont donc les billons qui fournissent la moyenne la plus élevée. L'augmentation par rapport au témoin relatif (écartement $40 \mathrm{~m}$ ) est de $60 \mathrm{p}$. cent.

Par rapport au témoin «absolu » (centre de la modalité $40 \mathrm{~m}$, moyenne $23,8 \mathrm{~cm}$ ) cette augmentation est de 62 p. cent.

Cependant, l'eau stagnant dans les creux de billons des blocs 3 et 4, il nous a paru intéressant d'effectuer une comparaison de moyennes pour les seuls blocs 1 et 2 .

Alors l'essai s'avère hautement significatif. 
Les billons fournissent de loin la moyenne la plus élevée. L'augmentation de croissance en hauteur par rapport aux $40 \mathrm{~m}$ est de $108 \%$, et, par rapport au centre de la modalité $40 \mathrm{~m}$, de 110 p. cent.

3.222. Évaux-et-Ménil (tableau 8).

\begin{tabular}{|c|c|c|c|c|c|c|c|c|}
\hline \multirow[b]{2}{*}{$\begin{array}{l}\text { Espèces } \\
\text { Species }\end{array}$} & \multirow[b]{2}{*}{$10 \mathrm{~m}$} & \multicolumn{7}{|c|}{$\begin{array}{l}\text { Longueur des pousses } 1969+1970 \text { à Évaux-et-Ménil (en cm) } \\
\text { Length of the shoots } 1969+1970 \text { at Évaux-et-Ménil (in cm) }\end{array}$} \\
\hline & & $20 \mathrm{~m}$ & $40 \mathrm{~m}$ & $\begin{array}{l}\text { Billons } \\
\text { Ridges }\end{array}$ & $\begin{array}{l}\text { F théorique } \\
\text { à } 5 \text { et } 1 \mathrm{p} \text {. cent } \\
\text { Theorical F } \\
\text { at } 5 \text { and } 1 \text { p. cent }\end{array}$ & $\begin{array}{c}\text { F calculé } \\
\text { calculated F }\end{array}$ & $\begin{array}{l}\text { P.P.D.S. } \\
\text { à } 5 \text { p. cent } \\
\text { L.S.D. } \\
\text { at } 5 \text { p. cent }\end{array}$ & $\begin{array}{l}\text { P.P.D.S. } \\
\text { à } 1 \text { p. cent } \\
\text { L.S.D. } \\
\text { at } 1 \text { p. cent }\end{array}$ \\
\hline $\begin{array}{l}\text { Épicéa ..... } \\
\text { Spruce }\end{array}$ & 13,6 & 13,3 & 12,1 & 14,3 & $3,86-6,99$ & 1,41 & & \\
\hline $\begin{array}{l}\text { Douglas.... } \\
\text { Douglas fir }\end{array}$ & 27,8 & 27,3 & 24,4 & 37,3 & $3,86-6,99$ & $6,26^{+}$ & 7.4 & 10,7 \\
\hline $\begin{array}{l}\text { Mélèze } \\
\text { Larch }\end{array}$ & 38,7 & 35,7 & 36,7 & 44,2 & $3,86-6,99$ & 1,87 & & \\
\hline
\end{tabular}

\section{Épicéa.}

L'essai n'est pas significatif. En fait, la pousse 1970 est très courte (de l'ordre de 5 à $7 \mathrm{~cm}$ ), pas plus grande que celle de 1969; le véritable « démarrage » de l'Épicéa n'a pas encore eu lieu, ce qui est bien normal. C'est pourquoi l'influence éventuelle des diverses modalités d'assainissement sur la croissance en hauteur n'a pu encore se manifester.

\section{Douglas.}

L'augmentation de croissance en hauteur provoquée par une plantation sur billons par rapport au témoin relatif (fossés à écartement $40 \mathrm{~m}$ ) est de $53 \mathrm{p}$. cent.

\section{Mélèze.}

Bien que l'essai ne soit pas significatif, on constate une différence de pousse assez sensible entre les billons et chacune des modalités «fossés " : l'augmentation de croissance en hauteur provoquée par une plantation sur billons par rapport au témoin relatif (fossés à écartement $40 \mathrm{~m}$ ) est de $20 \mathrm{p}$. cent.

\section{IV. - DISCUSSION ET CONCLUSION GÉNÉRALE}

\section{1. - Discussion des résultats obtenus sur billons à Essegney}

Nous avons signalé que la réalisation des billons dans les blocs 3 et 4 laissait à désirer : par suite d'un défaut de pente, l'eau stagne anormalement dans le creux de ces billons. 
Nous avons déjà comparé la croissance du Cyprès de Lawson suivant le traitement uniquement dans les blocs 1 et 2 : sa pousse qui était en moyenne la plus forte sur billons mais d'une façon non significative dans l'ensemble de l'expérience, l'était encore bien plus et très significativement lorsque l'on ne considérait que les blocs 1 et 2 .

On peut donc se demander si, pour les autres essences, les résultats ne seraient pas différents si l'on ne tenait compte que des blocs 1 et 2 et en particulier si la moyenne des pousses sur billons ne deviendrait pas supérieure à celle des modalités 20 ou $10 \mathrm{~m}$.

En ce qui concerne le taux de reprise des 3 essences concernées (Épicéa, Douglas et Mélèze), il est très bon dans les modalités 10 et $20 \mathrm{~m}$, donc le problème ne se pose pratiquement pas.

Quant à la croissance en hauteur, nous obtenons, en utilisant uniquement les blocs 1 et 2, les valeurs indiquées dans le tableau 9.

$$
\text { TABLEAU } 9 \text { - TABLE } 9
$$

Longueur des pousses $1969+1970$ dans les blocs $1+2$ à Essogney (en cm)

Length of the shoots $1969+1970$ in the blocks $1+2$, at Essegney (in cm)

\begin{tabular}{|c|c|c|c|}
\hline & $\begin{array}{l}\text { Épicéa } \\
\text { Spruce }\end{array}$ & $\begin{array}{l}\text { Douglas } \\
\text { Douglas fir }\end{array}$ & $\begin{array}{l}\text { Mélèze } \\
\text { Larch }\end{array}$ \\
\hline $\begin{array}{l}10 \mathrm{~m} \ldots \ldots \ldots \ldots \ldots \ldots \ldots \\
20 \mathrm{~m} \ldots \ldots \ldots \ldots \ldots \ldots \\
\text { Billons........... } \\
\text { Ridges }\end{array}$ & $\begin{array}{l}20,2 \\
20,0 \\
17,8\end{array}$ & $\begin{array}{l}44,5 \\
43,7 \\
36,3\end{array}$ & $\begin{array}{l}60,6 \\
46,4 \\
48,4\end{array}$ \\
\hline
\end{tabular}

On constate que la moyenne des pousses reste en général, sur billons, inférieure à ce qu'elle est dans les modalités 10 et $20 \mathrm{~m}$. La seule exception concerne la modalité $20 \mathrm{~m}$ pour le Mélèze; pour cette essence, la moyenne des pousses sur billons pour les blocs 1 et 2 est très supérieure à ce qu'elle est pour l'ensemble des quatre blocs.

De toute façon, pour chacune des trois essences, la moyenne des pousses sur billons reste inférieure à leur moyenne dans la meilleure modalité « fossés » et cela significativement.

En conséquence, les résultats essentiels présentés précédemment ne sont pas remis en cause.

On tentera néanmoins à l'avenir d'améliorer l'évacuation de l'eau pouvant stagner dans le creux des billons.

\section{2. - Synthèse des résultats d'Essegney}

Rappelons d'abord l'essentiel des résultats concernant l'assainissement du sol.

L'abaissement du niveau de la nappe est apparemment peu marqué avec un drainage à écartement de fossés de $40 \mathrm{~m}$. Il est très net par contre lorsqu'on passe à l'écartement $20 \mathrm{~m}$. 
La différence de niveau est bien plus faible entre les écartements $20 \mathrm{~m}$ et $10 \mathrm{~m}$ qu'entre les écartements $40 \mathrm{~m}$ et $20 \mathrm{~m}$. Le niveau moyen de la nappe pour la modalité billons est intermédiaire entre ceux des écartements $40 \mathrm{~m}$ et $20 \mathrm{~m}$.

L'effet des travaux de sol sur le taux de reprise et la croissance en hauteur des plants est plus ou moins marqué suivant les essences. Lorsqu'il se produit, il est en liaison étroite avec l'abaissement relatif de la nappe, sauf pour le Cyprès de Lawson.

L'assainissement ne semble être d'aucune utilité jusqu'à présent pour le Pin Weymouth : il n'améliore pas sa croissance en hauteur et son taux de reprise est très bon même en l'absence d'assainissement.

Pour l'Épicéa, le Douglas et le Mélèze, un drainage par fossés assez rapprochés s'avère nettement plus efficace qu'une plantation sur billons. Il améliore nettement la croissance de l'Épicéa ainsi que le taux de reprise et la croissance du Douglas et du Mélèze. Un écartement de fossés de $20 \mathrm{~m}$ a des conséquences pratiquement aussi favorables qu'un écartement de $10 \mathrm{~m}$ : il s'avère donc suffisant pour le moment.

Pour le Cyprès de Lawson par contre, il n'y a pas parallélisme entre abaissement de la nappe et comportement des plants : la plantation sur billons s'avère le traitement de loin le plus efficace, tant pour la reprise que pour la croissance. Il est possible que cet effet favorable des billons ne soit pas la conséquence seulement de l'assainissement qui en découle, mais que d'autres facteurs interviennent également (épaisseur de l'horizon organique, stabilité structurale et autres propriétés physiques, etc.).

\section{3. - Synthèse des résultats d'Évaux-et-Ménil et comparaison des deux expériences}

Les différences de niveau de nappe entre les trois modalités fossés sont très peu marquées, ce qui tend à montrer la faible efficacité du drainage par fossés à Évaux. Par contre sur sommets de billons, l'épaisseur de sol assaini est sans doute bien supérieure à ce qu'elle est dans les modalités fossés.

L'assainissement agit sur le comportement des plants plus ou moins suivant les essences. Mais lorsque son effet se manifeste, il est comme à Essegney, en relation étroite avec l'épaisseur de sol assaini.

Pour l'Épicéa, aucun type d'assainissement ne modifie le comportement des plants. La plantation sur billons améliore la reprise et la croissance du Mélèze (mais les différences ne sont pas significatives jusqu'à présent) et surtout du Douglas.

Si l'on compare les résultats obtenus à Essegney et à Évaux pour les trois essences communes, on constate que dans les deux expériences, du moins jusqu'à présent, l'assainissement s'est avéré plus efficace pour le Mélèze que pour l'Épicéa et pour le Douglas que pour le Mélèze. Mais dans l'ensemble, si la meilleure modalité amène un taux de reprise tout à fait convenable tant à Évaux qu'à Essegney, la pousse correspondante est plus forte à Essegney, de 51 p. cent pour l'Épicéa, 27 p. cent pour le Mélèze et 17 p. cent pour le Douglas.

\section{4. - Conclusion}

Les résultats obtenus à la fin des deux premières années sont évidemment susceptibles de varier considérablement à l'avenir, tant dans un sens d'accroissement de l'efficacité de 
l'assainissement que dans le sens inverse. Il ne serait pas étonnant en particulier que très prochainement se produise une différence significative de croissance entre traitements pour l'Épicéa à Évaux et le Pin Weymouth à Essegney : en effet, leur véritable « démarrage » en hauteur n'avait pas encore eu lieu lors des dernières mesures.

Néanmoins les résultats enregistrés jusqu'à présent ont un intérêt propre en raison de la grande importance pratique que revêt la manière dont une plantation s'installe et démarre. Ils sont très encourageants car, du moins pour les traitements les plus efficaces, les plants se comportent d'une façon comparable à ce qui se produit sur des sols réputés bien plus favorables, tant en ce qui concerne le taux de reprise que pour la croissance en hauteur. Les résultats les plus intéressants concernent le Douglas, le Mélèze et le Cyprès de Lawson à Essegney et le Douglas à Évaux-et-Ménil; personne n'aurait risqué d'introduire ces essences, du moins à une échelle importante, sur sol très hydromorphe. Or les premiers résultats permettent un certain optimisme, particulièrement en ce qui concerne le Douglas. Ainsi, à Essegney après drainage, cette essence a une pousse initiale deux fois plus importante que celle de l'Épicéa; à Évaux l'assainissement permet de réduire considérablement la mortalité qui se produit au cours des deux premières années et qui ne peut que faire déconseiller cette essence sans travail du sol préalable.

Il est certain que la réalisation de ces travaux d'assainissement augmente substantiellement l'investissement de départ. Si l'on se fonde sur le coût des opérations effectuées à Essegney et Évaux-et-Ménil, on aboutit, pour un réseau de fossés à écartement de $20 \mathrm{~m}$, à un prix de revient de $800 \mathrm{~F}$ par hectare et pour un billonnage sur toute la surface, à $450 \mathrm{~F}$ par hectare, chiffre qui peut être sensiblement augmenté si un labour préalable s'avère indispensable. Cependant, dans la mesure où ces techniques se développeront, on peut espérer une diminution du coût unitaire.

De toute façon, le bilan économique est évidemment impossible à dresser à l'heure actuelle; on ne peut savoir s'il sera finalement positif. Il est fort possible qu'en plus de l'augmentation de la production courante, l'assainissement du sol diminue les risques de chablis, donc permette de maintenir le peuplement en place plus longtemps, et cela devra intervenir dans le bilan.

Par ailleurs, l'assainissement peut s'avérer utile sur un plan autre que celui de la rentabilité économique à court terme. En effet, plusieurs travaux ont contribué à mettre en lumière le risque de dégradation du sol à plus ou moins longue échéance qu'amène une monoculture d'Épicéa sur sol hydromorphe. L'utilisation de techniques d'assainissement, permettant d'introduire des essences plus délicates, mais moins dégradantes, tout en assurant une production convenable, peut se placer dans le cadre de la préservation des équilibres biologiques naturels.

Les deux expériences d'Essegney et d'Évaux-et-Ménil seront complétées par des essais analogues situées dans des conditions écologiques un peu diférentes et où seront peut-être testées d'autres essences résineuses intéressantes telles Abies grandis ou Thuya plicata.

Reçu pour publication en juin 1972. 


\section{REMERCIEMENTS}

Nous tenons à remercier Messieurs Mormiche, alors Chef du Centre de Gestion de l'O.N.F. d'Épinal-Nord et STOQUert, Chef du Cantonnement d'Épinal-Charmes - grâce à leur compréhension et à leur aide, ces expériences ont pu être implantées - , ainsi que Messieurs DuC et MARCHAL, de la Chambre d'Agriculture des Vosges qui ont accepté de faire exécuter les travaux malgré des conditions naturelles difficiles.

\section{SUMMARY}

\section{INITIAL RESULTS OF TWO DRAINAGE EXPERIMENTS WITH PLANTATION OF CONIFERS}

Important forest surfaces have in the N.E. of France soils with pseudogley. The production of the natural stand of hardwoods gives little income; then conifers are often planted on these soils. Often Norway Spruce and sometimes White Pine are used; but it may be asked if other species would not give a more interesting production, at any rate after previous drainage of the soil.

One of the experiments (Essegney) is placed on typical pseudogley (table $\mathrm{n}^{\circ} 1$ ) formed on old alluvial sediments covered with loam; the relatively impermeable layer begins at about -45 to $-50 \mathrm{~cm}$. The soil of the second one is a pelosol-pseudogley formed on clayed keuper (table $\left.\mathrm{n}^{\circ} 2\right)$; its impermeable layer begins between -25 and $-30 \mathrm{~cm}$. The climate is identical for the two experiments. The slope, very small at Essegney, varies from 2 to $5 \%$ at Evaux.

The species tried at Essegney are Norway Spruce, Douglas fir, European Larch, White Pine and Lawson's Cypress. Only the first three one were planted at Evaux.

Four drainage modalities were compared : draining ditches distant of about $40 \mathrm{~m}, 20 \mathrm{~m}$ and $10 \mathrm{~m}$, and ridges of $2,30 \mathrm{~m}$ of breadth. Before that, a crossed ploughing was made on the whole surface. Four blocks were settled. The results presented here are those recorded two years after plantation.

Here are the results obtained at Essegney :

- Lowering of the water-table (recorded in piezometers) (figure 1 and table 3 ) is small when ditches are distant of $40 \mathrm{~m}$. On the other hand it is very marked for the modality $20 \mathrm{~m}$. The difference of level is very smaller between the modalities 20 and $10 \mathrm{~m}$. than between the modalities 40 and $20 \mathrm{~m}$. The average level of the water-table for the ridges is intermediate between those of modalities with ditches distant of 40 and $20 \mathrm{~m}$.

- The influence of soil works on lowering of mortality rate (table 5) and on the growth in height (table 7) of the plants is more or less important according to the species. When an influence appears, it is very bounded with the relative lowering of the water-table level, except for the Lawson's Cypress.

- The drainage seems to be without usefulness up to now for the White Pine : it does not improve its growth in height and its mortality is of very little importance even without drainage.

- For Spruce, Douglas Fir and Larch, a drainage by near enough ditches is more efficient than the ridges. It improves clearly the growth of Spruce, Douglas Fir and Larch and reduces the mortality of Douglas Fir and Larch. The modality $20 \mathrm{~m}$ is practically as favorable as the modality $10 \mathrm{~m}$ : it is therefore sufficient at least up to now.

- For Lawson's cypress on the contrary, there is no parallelism between lowering of watertable and behaviour of the plants : plantation upon ridges is clearly the most efficient modality for their survival as for their growth.

At Evaux-et-Menil, the results are :

- the differences of level of the water-table (table 4) between the three modalities with ditches are very small : that shows the little efficience of the drainage with ditches at Evaux. On the other hand, the relative level of the water-table on tops of the ridges is without doubt clearly lower than in the modalities with ditches.

- the drainage influences the behaviour of the plants more or less according to the species (tables 6 and 8). When an influence appears, it is, as at Essegney, very bounded with the relative lowering of the water-table level. 
- for Spruce, not any modality of drainage does change the behaviour of the plants. The plantation upon ridges improves the survival rate and the growth of Larch (but the differences are not significant up to now) and chiefly of Douglas fir (its mortality rate, for instance, decreases from 40 to $6 \mathrm{p}$. cent).

- comparing the results obtained at Essegney and Evaux for the best modality (for the three common species), it is seen that, if the survival rate is quite suitable in both experiments, the corresponding shoot is larger at Essegney than at Evaux (of 51 p. cent for Spruce, 17 p. cent for Douglas fir and $27 \mathrm{p}$. cent for Larch).

These results are very encouraging, though they may much vary in the future; they have an own interest because the great practical importance of the way in which a plantation survives and starts.

\section{ZUSAMMENFASSUNG}

\section{ERSTE ERGEBNISSE VON ZWEI ENTWASSERUNGSVERSUCHEN IN NADELHOLZPFLANZUNGEN}

Auf zwei verschiedenen Standorten wurde der Einfluss von vier verschiedene Meliorationvarianten (Entwässerungsgräben in 40, 20 und $10 \mathrm{~m}$ Abstand sowie Bifangstreifen von ca $0,5 \mathrm{~m}$ Höhe und $2,5 \mathrm{~m}$ Breite) auf das Wachstum und den Pflanzausfall verschiedener Nadelhölzer untersucht. Die vorliegenden Ergebnisse betreffen die ersten zwei Jahre nach der Pflanzung.

Versuch " Essegney» :

Standort : Lehmüberdeckter Pseudogley auf Moselalluvium.

Holzarten : Fichte, Douglasie, Strobe, europäische Lärche und Chamaecyparis.

Ergebnisse : Die Entwässerungsgräben in 20 und $10 \mathrm{~m}$ Abstand erbrachten eine bemerkenswerte Absenkung des Stauwasserspiegels, wodurch das Höhenwachstum der Fichte und die Uberlebensprozente und das Wachstum von Douglasie und Lärche bedeutend verbessert wurden. Bei Chamaecyparis zeigten jedoch die Bifangstreifen die besten Erfolge.

Versuch « Evaux et Ménil :

Standort : Pelosol-Pseudogley mit 25 bis $30 \mathrm{~cm}$ hoher Staublehmüberdeckung auf Keupermergel.

Holzarten : Fichte, Douglasie und Lärche.

Ergebnisse : Nur die Bifangstreifen ermöglichten eine Absenkung des Stauwasserspiegels, welche sich auf den Pflanzerfolg und das Höhenwachstum der Douglasie äusserst günstig auswirkte.

\section{RÉFÉRENCES BIBLIOGRAPHIQUES}

Befrs W. FJ. VAn, 1964. La méthode du trou à la tarière. Terres et Eaux, no 44, 16-28.

Guyon G., 1970. Le drainage des nappes perchées. Bull. techn. Génie rural, no 102, janvier, 1-51.

LÉvy G., 1969. Premiers résultats d'étude comparée de la nappe temporaire des pseudogleys sous résineux et sous feuillus. Ann. Sci. for., 26 (1), 65-79.

Poirée M., Ollier Ch., 1965. Assainissement agricole. Éditions Eyrolles, Paris, 444 pages.

Pourtet J., 1964. Les repeuplements artificiels. École nationale des Euax et Forêts, Nancy, 278 pages.

Schlenker G. et al., 1969. Untersuchungen über die Auswirkungen des Fichtenreinanbaus auf Parabraunerden und Pseudogleye des Neckarlandes. Mitt. des Vereins für Forst. Stand. u. Forstpfl. heft 19, okt., 72-114.

Vessereau A., 1960. Méthodes statistiques en hiologie et en agronomie. J.-B. Baillière et fils, Paris, 540 pages. 